\title{
Fuzzy Torque Control of the Bionic Flexible Manipulator Actuated by Pneumatic Muscle Actuators
}

\author{
Kai Liu*, Yining Chen, Jiaqi Xu, Yang Wu, Yonghua Lu and Dongbiao Zhao
}

\begin{abstract}
A bionic flexible manipulator driven by pneumatic muscle actuator (PMA) can better reflect the flexibility of the mechanism. Current research on PMA mainly focuses on the modeling and control strategy of the pneumatic manipulator system. Compared with traditional electro-hydraulic actuators, the structure of PMA is simple but possesses strong nonlinearity and flexibility, which leads to the difficulty in improving the control accuracy. In this paper, the configuration design of a bionic flexible manipulator is performed by human physiological map, the kinematic model of the mechanism is established, and the dynamics is analyzed by Lagrange method. A fuzzy torque control algorithm is designed based on the computed torque method, where the fuzzy control theory is applied. The hardware experimental system is established. Through the co-simulation contrast test on MATLAB and ADAMS, it is found that the fuzzy torque control algorithm has better tracking performance and higher tracking accuracy than the computed torque method, and is applied to the entity control test. The experimental results show that the fuzzy torque algorithm can better control the trajectory tracking movement of the bionic flexible manipulator. This research proposes a fuzzy torque control algorithm which can compensate the error more effectively, and possesses the preferred trajectory tracking performance.
\end{abstract}

Keywords: Pneumatic muscle actuator, Bionic flexible manipulator, Computed torque method, Fuzzy torque control

\section{Introduction}

The pneumatic muscle actuator (PMA) is a new pneumatic element with characteristics similar to biological muscles. In addition to the low cost, cleanliness, and other advantages of pneumatic transmission technology, it also possesses the characteristics of simple structure, high power/weight ratio, good flexibility, and so on. PMA is one of the typical representatives of new pneumatic components since the development of pneumatic technology. Because of its extremely similar function with human muscle, PMA has been applied to robotic arms [1], robotic lower limbs [2, 3] and robotic claws [4, 5]. It is expected to have great potential applications in physiotherapy [6], vehicles [7], robots [8, 9] and other fields. PMA has become the new research hotspot in robotics.

\footnotetext{
*Correspondence: liukai@nuaa.edu.cn

College of Mechanical and Electrical Engineering, Nanjing University

of Aeronautics and Astronautics, Nanjing 210016, China
}

The characteristics and models of PMA are the basis of control applications. Although the structure is very simple, it is very difficult to model accurately owing to the influence of nonelastic deformation and friction; the model of PMA is a typical a nonlinear model. In a previous study [10], we established the PMA mechanics model through neural network and achieved good results.

Because of the nonlinearity and flexibility of PMA, and the compressibility of the air, the control strategy is a hot topic in pneumatic muscle research. Since research on related manipulators driven by fluid transmission actuators have yielded good results [11-13], different control methods have been proposed to improve the control effect of the pneumatic manipulator system. Carbonell et al. [14] designed a fuzzy backstepping controller by considering the nonlinearity characteristics of PMAs, in which the system controlled has global convergence and certain robustness. Zhu et al. [15] proposed an adaptive robust attitude controller to solve the uncertainty and 
nonlinearity in the dynamics of a parallel robot driven by PMAs to achieve accurate attitude tracking and reduce control input jitter. Shen et al. [16] designed a hybrid sliding mode controller with an adaptive fuzzy cerebellar model articulation controller for the online approximation of a spring mass position control system driven by PMA. Lin et al. [17] simplified PMA into a three-element model. Considering that the $\mathrm{H} \infty$ robust controller has superior ability to handle system uncertainty, an $\mathrm{H} \infty$ robust controller was designed to ensure the stability and superior performance of the PMA system. Ba et al. [18] proposed an advanced position tracking control method for the control of PMA system. The neural network and sliding mode control algorithm were integrated into the new robust controller to guarantee the fast response, high accuracy, and robustness of the system. Wang et al. [19] established a cascade human elbow joint model driven by PMAs, and designed a fuzzy PI controller for positiontracking experiments to improve the control accuracy and verify the effectiveness of the fuzzy controller. Zhao et al. [20] proposed a self-adaptive disturbance rejection control strategy to improve the control effects of the single-joint manipulator driven by PMAs, and effectively solve nonlinear problems of the manipulator control system. Although these control theories improve the control effect of the pneumatic system to some extent, there are still many limitations such as model dependence, poor anti-interference ability, poor control precision, nonlinearity and uncertainty of the system, and so on.

More and more attention is being paid to the study of PMAs in the humanoid arm [21]. Hence, a humanoid arm mechanism driven by a PMA group based on the superior characteristics of PMA is proposed, and further research on the control strategy is carried out. Firstly, according to the analysis of the physiological characteristics of the human upper arm, the configuration design of the bionic flexible manipulator of serial parallel mechanism is realized, and the kinematic and dynamic model of the mechanism is established. The fuzzy torque controller is designed based on the fuzzy controller [22], and the application of computed torque method to test the trajectory tracking of the flexible manipulator is realized as well.

\section{Characteristic Analysis of Bionic Flexible Manipulator}

\subsection{Configuration Analysis}

The joint robot, which has the advantages of simple structure, small occupied space, large relative working space, etc., is one of the most widely used robots $[23,24]$. Generally speaking, the arrangement of joints actuated by a humanoid arm can be divided into two categories: PMA serial mechanism and PMA parallel mechanism. For example, the study of the 7-DOF manipulator actuated by PMA was implemented [25] from the perspective of functional bionics, and the PMA actuated antagonistic bionic joint was designed [26]; a 3-DOF parallel robot platform based on PMA was proposed [27], and a 10-DOF robot arm driven by PMA group was designed [1]. The humanoid shoulder joint is usually designed as a series of rotating joints in order to realize the bionic structure. However, the analysis of this design is not based on the basic structure of the joint. The movement of the human shoulder can be divided into two parts: (a) the movement of the upper body and scapula; and (b) the movement of the humerus and scapula.

The skeleton of the human shoulder joint consists mainly of the shoulder blade, clavicle, and humerus. The shoulder blade has six kinds of motion including lift, inhibition, internal rotation, external rotation, abduction, and adduction. Relative to the shoulder blade, the humerus has six kinds of motion including flexion, extension, internal rotation, external rotation, abduction, and adduction [28].

From Table 1, the number of muscles that drive the motions of the shoulder blade and humerus is larger and the position structures of these muscles are more complex. Thus, muscles that have a greater impact on shoulder joint motion are only simulated by PMAs in the structural design. As shown in Figure 1, the trapezius, levator scapulae, serratus anterior, and latissimus dorsi are muscles that have a greater impact on shoulder blade motion; and four

Table 1 Motions of shoulder joint

\begin{tabular}{|c|c|c|c|}
\hline Scapular joint & & Glenohumeral joint & \\
\hline Lift & Trapezius, Levator scapulae, Rhomboideus & Flexion & Deltoid, Pectoralis major, Coracobrachialis \\
\hline Inhibition & Trapezius, Serratus anterior, Latissimus dorsi & Extension & Deltoid, Latissimus dorsi, Teres major \\
\hline Internal rotation & Trapezius, Serratus anterior & Internal rotation & Subscapularis, Deltoid \\
\hline External rotation & $\begin{array}{l}\text { Levator scapulae, Rhomboideus, Pectoralis minor, Latis- } \\
\text { simus dorsi }\end{array}$ & External rotation & Infraspinatus, Teres minor, Deltoid \\
\hline Abduction & Serratus anterior, Pectoralis minor & Abduction & Supraspinatus, Deltoid \\
\hline Adduction & Trapezius, Rhomboideus, Latissimus dorsi & Adduction & Latissimus dorsi, Pectoralis major \\
\hline
\end{tabular}




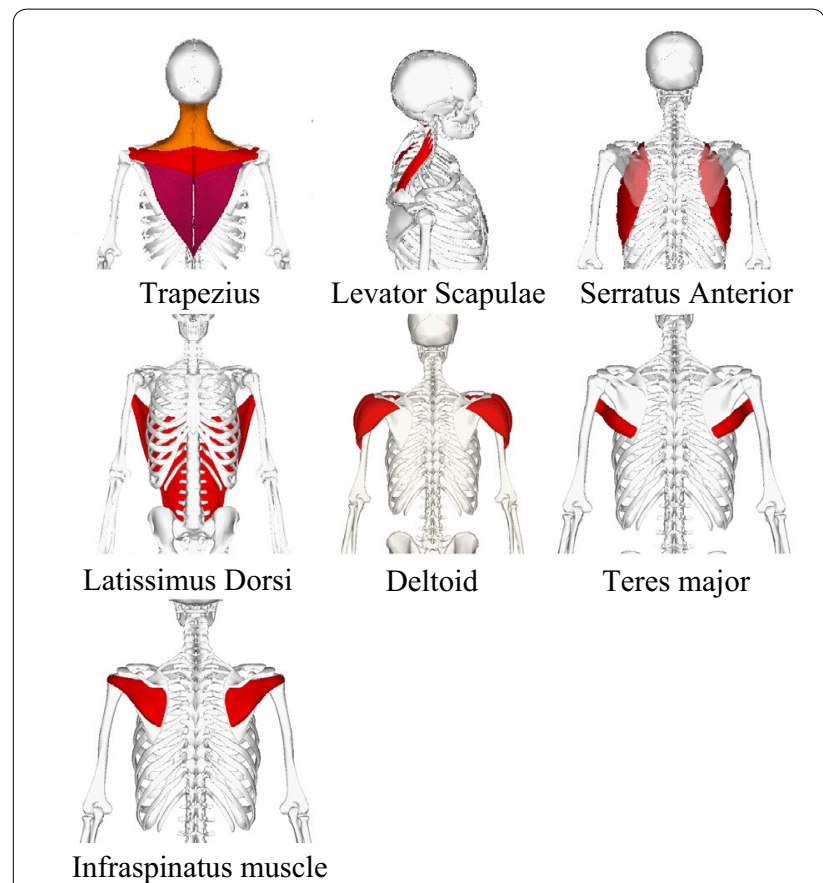

a Partial muscles of the shoulder joint

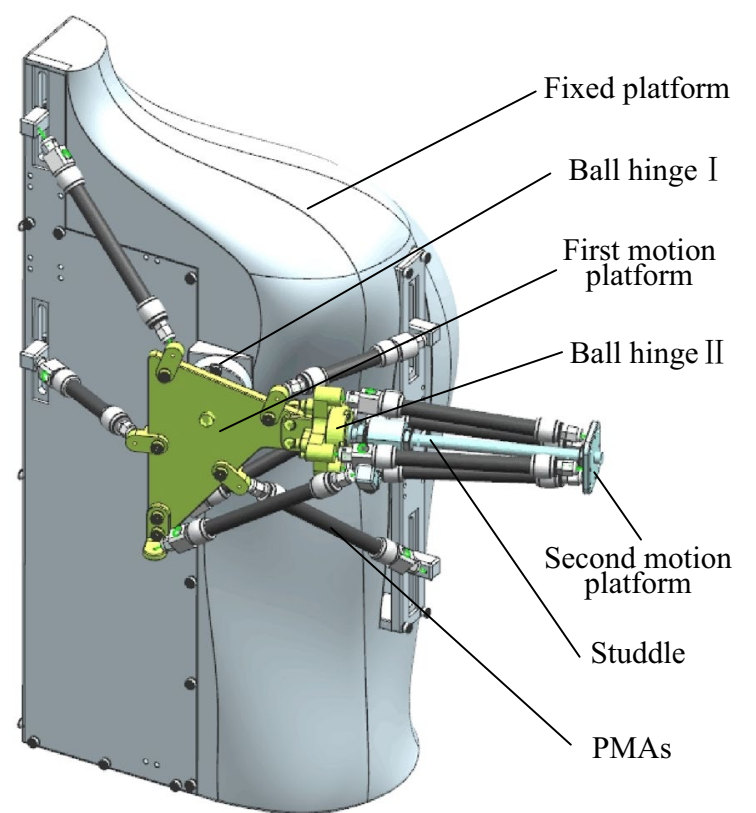

b Three-dimensional model of the shoulder joint

Figure 1 Structural design of the shoulder joint

PMAs $\left(\mathrm{P}_{1}-\mathrm{P}_{4}\right)$ were used for the bionic driving. The deltoid, teres major, and infraspinatus muscle are muscles that have a greater impact on humerus motion, and six PMAs $\left(\mathrm{P}_{5}-\mathrm{P}_{10}\right)$ were used for the bionic driving [29].
The connecting part of the human shoulder joint is divided into two parts. One is the connection between the upper torso and the shoulder blade. There is a scapulothoracic joint between the shoulder and the upper torso in the human body. In the design, a ball hinge was used for the bionic connection to achieve the shoulder blade motion. The other is the connection between the humerus and the shoulder blade. The glenohumeral joint, which consists of the humeral head and scapula glenoid, is a typical and most flexible ball-and-socket joint [30]. It connects the humerus and shoulder blade, and was simulated by another ball hinge for the bionic connection in the design.

\subsection{Kinematics and Dynamics Analysis}

The schematic diagram and coordinate system of the bionic shoulder joint are set up as shown in Figure 2. The coordinate origin $O_{1}$ of the fixed coordinate system $O_{1} X_{1} Y_{1} Z_{1}$ is located at the ellipse center at the bottom of the trunk; $O_{1} X_{1}$ is along the long axis of the ellipse; and $O_{1} Y_{1}$ is along the short axis of the ellipse. The coordinate origin $\mathrm{O}_{2}$ of the relative coordinate system $\mathrm{O}_{2} \mathrm{X}_{2} Y_{2} Z_{2}$ is located at the rotation center of ball hinge I, and the other axes are the same as the fixed coordinate system. The initial position of the relative coordinate system $\mathrm{O}_{3} X_{3} Y_{3} Z_{3}$ is the same as the coordinate system $\mathrm{O}_{2} X_{2} Y_{2} Z_{2}$, and the coordinate origin $O_{3}$ is located at the rotation center of ball hinge II. The initial position of the execution end coordinate system $O_{4} X_{4} Y_{4} Z_{4}$ is the same as that of coordinate system $\mathrm{O}_{3} X_{3} Y_{3} Z_{3}$, and the coordinate origin $\mathrm{O}_{4}$ is located at the rotation center of the execution end.

Let the $X-Y-Z$ Euler angle $\left(\alpha_{1}, \beta_{1}, \gamma_{1}\right)$ be the rotation of the shoulder blade relative to the upper torso; ${ }_{2}^{1} \boldsymbol{T}$ be the transformation matrix from $O_{2} X_{2} Y_{2} Z_{2}$ to $O_{1} X_{1} Y_{1} Z_{1}$; the $X-Y$-Z Euler angle $\left(\alpha_{2}, \beta_{2}, \gamma_{2}\right)$ be the rotation of the humerus relative to the shoulder blade; ${ }_{3}^{2} T$ be the transformation matrix from $\mathrm{O}_{3} X_{3} Y_{3} Z_{3}$ to $\mathrm{O}_{2} X_{2} Y_{2} Z_{2} ;{ }_{4}^{3} \boldsymbol{T}$ be the transformation matrix from $O_{4} X_{4} Y_{4} Z_{4}$ to $O_{3} X_{3} Y_{3} Z_{3}$; and ${ }_{4}^{1} \boldsymbol{T}$ be the transformation matrix from the execution end to the fixed coordinate system.

$$
{ }_{4}^{1} \boldsymbol{T}={ }_{2}^{1} \boldsymbol{T}\left(\alpha_{1}, \beta_{1}, \gamma_{1}\right){ }_{3}^{2} \boldsymbol{T}\left(\alpha_{2}, \beta_{2}, \gamma_{2}\right){ }_{4}^{3} \boldsymbol{T} .
$$

In Figure 2(a), one end of PMA that drives the first motion platform is connected to the fixed platform and its coordinate in $O_{1} X_{1} Y_{1} Z_{1}$ is $\boldsymbol{A}_{i}$. Another end is connected to the first motion platform and its coordinate in $\mathrm{O}_{2} \mathrm{X}_{2} Y_{2} Z_{2}$ is $\boldsymbol{B}_{i}$. The length of four PMAs is

$$
L_{i}=\left|{ }_{2}^{1} \boldsymbol{R} \boldsymbol{B}_{i}+\boldsymbol{O}_{2}-\boldsymbol{A}_{i}\right|, \quad i=1-4,
$$

where ${ }_{2}^{1} \boldsymbol{R}$ is the attitude matrix of the first motion-platform. 


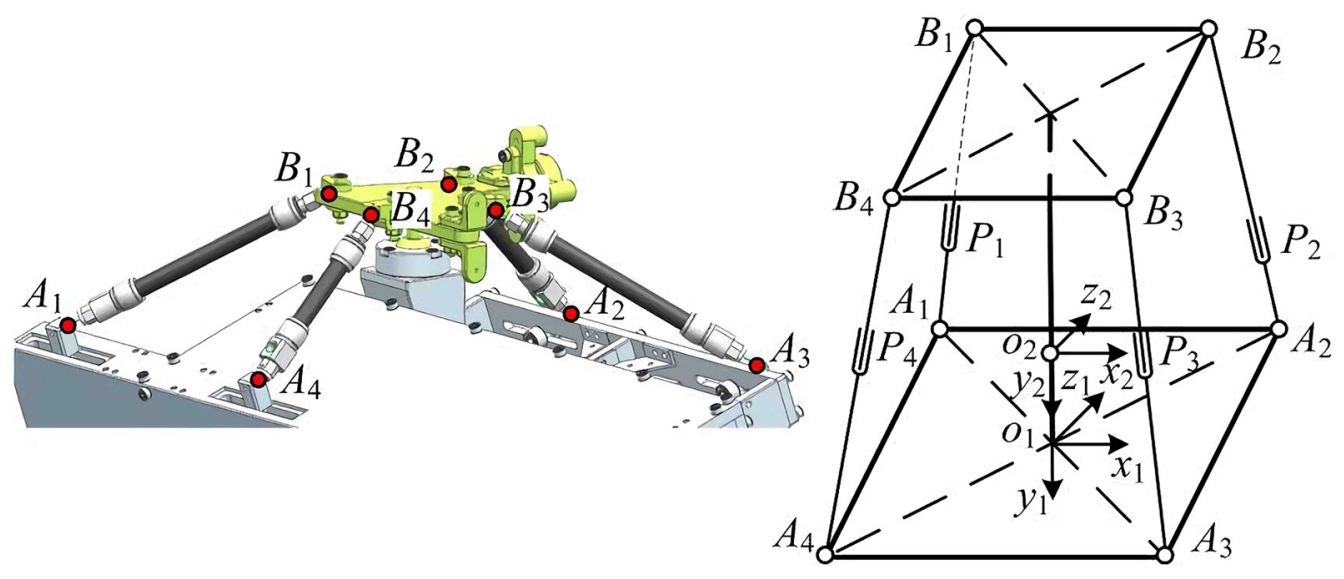

a Connection diagram of the upper torso and shoulder blade
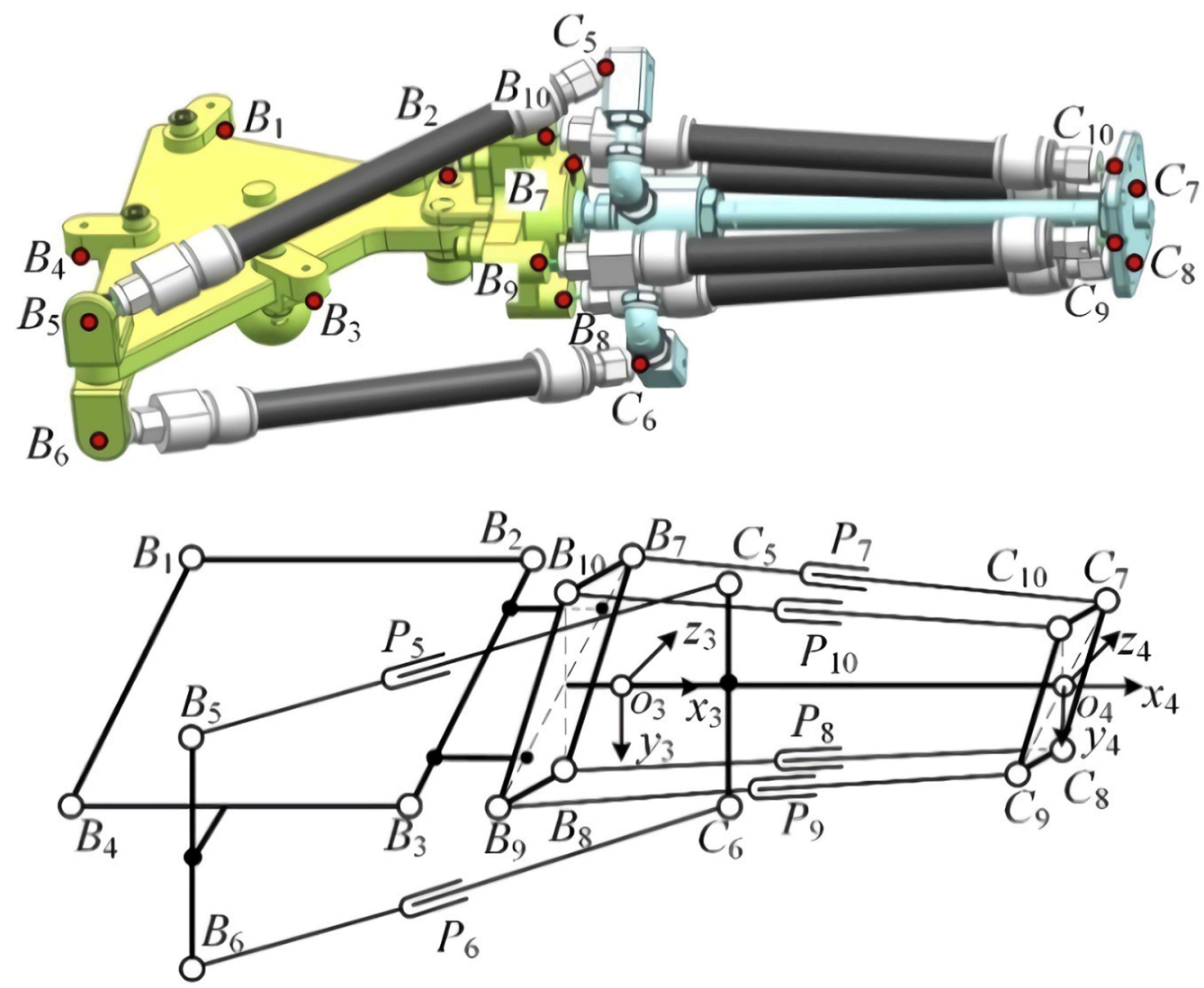

b Connection diagram of the humerus and shoulder blade

Figure 2 Simplified mechanism and coordinate systems of the shoulder joint

In Figure 2(b), one end of PMA that drives the second motion-platform is connected to the first motion-platform, and its coordinate in $\mathrm{O}_{2} \mathrm{X}_{2} Y_{2} Z_{2}$ is $\boldsymbol{B}_{i}$. Another end is connected to the second motion-platform and its coordinate in $\mathrm{O}_{3} X_{3} Y_{3} Z_{3}$ is $C_{i}$. The length of six PMAs is

$$
L_{i}=\left|{ }_{3}^{2} \boldsymbol{R} C_{i}+\boldsymbol{O}_{3}-\boldsymbol{B}_{i}\right|, \quad i=5-10,
$$


where ${ }_{2}^{3} \boldsymbol{R}$ is the attitude matrix of the second motion-platform.

The Lagrange method is used to establish the dynamic equation of the flexible manipulator. The Lagrange method is known as

$$
L_{\mathrm{E}}=K_{\mathrm{E}}-P_{\mathrm{E}},
$$

where $K_{\mathrm{E}}$ is the system's kinetic energy, $P_{\mathrm{E}}$ is the system's potential energy, and $\boldsymbol{q}$ is the generalized coordinate vector of the system. The following relationship is established:

$$
\frac{\mathrm{d}}{\mathrm{d} t}\left(\frac{\partial L_{\mathrm{E}}(\boldsymbol{q}, \dot{\boldsymbol{q}})}{\partial \dot{\boldsymbol{q}}}\right)-\frac{\partial L_{\mathrm{E}}(\boldsymbol{q}, \dot{\boldsymbol{q}})}{\partial \boldsymbol{q}}=\boldsymbol{\tau},
$$

where $\boldsymbol{\tau}$ is the generalized force (torque) vector acting on the generalized coordinates. The RPY angle is used as the generalized coordinates of the system, which is $\boldsymbol{q}=[\alpha \beta$ $\gamma]^{\mathrm{T}}$. The following expression is obtained by Eq. (5):

$$
\boldsymbol{M}(\boldsymbol{q}) \ddot{\boldsymbol{q}}+\boldsymbol{V}(\boldsymbol{q}, \dot{\boldsymbol{q}}) \dot{\boldsymbol{q}}+\boldsymbol{G}(\boldsymbol{q})=\boldsymbol{\tau},
$$

where $\boldsymbol{M}(\boldsymbol{q})$ is the inertia matrix of the system, $\boldsymbol{V}(\boldsymbol{q}, \dot{\boldsymbol{q}})$ is the Coriolis force and centrifugal force of the system, and $\boldsymbol{G}(\boldsymbol{q})$ is the gravity term.

It should be noted that $\boldsymbol{\tau}$ is not the output force of PMA, but the generalized torque acting on the generalized coordinates $\boldsymbol{q}$. The relationship between them is

$$
\dot{\boldsymbol{l}}=\boldsymbol{J} \dot{\boldsymbol{q}},
$$

where $\boldsymbol{J}$ is the Jacobi ratio of the corresponding joint, which characterizes the relationship between the aerodynamic speed of PMA and the angular velocity of the corresponding platform. According to the principle of virtual work, Eq. (8) is obtained:

$$
\boldsymbol{F}=\left(\boldsymbol{J}^{\mathrm{T}}\right)^{-1} \cdot \boldsymbol{\tau}=\left(\boldsymbol{J}^{\mathrm{T}}\right)^{-1} \cdot[\boldsymbol{M}(\boldsymbol{q}) \ddot{\boldsymbol{q}}+\boldsymbol{V}(\boldsymbol{q}, \dot{\boldsymbol{q}})+\boldsymbol{G}(\boldsymbol{q})],
$$

where $\boldsymbol{F}$ is the force vector consisting of the output forces of PMA $i(i=1-10)$.

\subsection{Joint Trajectory Planning of Serial Mechanism}

The bionic flexible manipulator is a typical 6-DOF seriesparallel mechanism. Its end position space and attitude space are highly coupled. In this section, in order to smoothen the strong coupling mechanism trajectory tracking, based on the global optimization and parallel algorithm of the genetic algorithm, the smooth trajectory curves of each joint were obtained under a certain position trajectory curve of the end. The bionic flexible manipulator consists of ball hinge I and II, which constitute 6-DOF of bionic flexible manipulator; hence, the optimal variables are described as follows:

$$
x=\left\{\alpha_{1}, \beta_{1}, \gamma_{1}, \alpha_{2}, \beta_{2}, \gamma_{2}\right\} .
$$

Since the genetic algorithm is based on the fitness function of the required problem, the solution of the fitness function is very important. Assuming that the actual position of the end is $\left\{p x_{\mathrm{d}}, p y_{\mathrm{d}}, p z_{\mathrm{d}}\right\}$, the ideal position is $\{p x, p y, p z\}$. The first objective is to minimize the difference between the actual position and desired position:

$$
\min f_{1}(x)=\left|p x_{\mathrm{d}}-p x\right|+\left|p y_{\mathrm{d}}-p y\right|+\left|p z_{\mathrm{d}}-p z\right| .
$$

Suppose the estimated rotation angle at a certain time is $x_{k}=\left\{\alpha_{1 k}, \beta_{1 k}, \gamma_{1 k}, \alpha_{2 k}, \beta_{2 k}, \gamma_{2 k}\right\}$, when the actual rotation angle is $x_{k-1}=\left\{\alpha_{1(k-1)}, \beta_{1(k-1)}, \gamma_{1(k-1)}, \alpha_{2(k-1)}, \beta_{2(k-1)}, \gamma_{2(k-1)}\right\}$. The secondary objective is the minimum value of the sum of the difference between the predicted rotation angle of hinge I and II at a certain moment relative to the actual rotation angle at the previous moment; hence, the joint trajectory curve can be relatively smooth.

$$
\begin{aligned}
\min f_{2}(x)= & \sum_{i=1}^{2}\left(\left|\alpha_{i k}-\alpha_{i(k-1)}\right|+\left|\beta_{i k}-\beta_{i(k-1)}\right|\right. \\
& \left.+\left|\gamma_{i k}-\gamma_{i(k-1)}\right|\right) .
\end{aligned}
$$

The primary goal of trajectory tracking is to ensure the reliability of the position curve. The secondary objective is to ensure the smoothness of the joint curve. By weighting the two objective functions with proper weights, the following objective function can be obtained:

$$
f_{f i t}=\min \left(0.9 f_{1}(x)+0.1 f_{2}(x)\right) .
$$

Let the ideal circular trajectory of the end be

$$
\left\{\begin{array}{l}
p x=p x_{0}-15 \\
p y=p y_{0}+50 \cos (w t), \\
p z=p z_{0}+50 \sin (w t) .
\end{array}\right.
$$

The size of the population is 100 . After 241 generations, the trajectory curve of the end and optimal trajectory curves of all joints are shown in Figure 3 and Figure 4. Although the tracking effect is good, there exists a trajectory jitter of each joint, which is not conducive to control.

To facilitate control, the joint trajectory curve must be smooth. Therefore, to improve the smoothness of the joint trajectory, MATLAB CFTOOL was used to fit the joint trajectory, as shown in Figure 5. Although there exists a larger error between the actual and ideal trajectory, the maximum position error of the $X$-axis, $Y$-axis, and $Z$-axis direction is only $1.536 \mathrm{~mm}, 1.816 \mathrm{~mm}$, and $0.958 \mathrm{~mm}$, respectively. Compared with the trajectory circle with a radius of $50 \mathrm{~mm}$, the error is smaller and the precision is satisfied. The curve is smoother, so the fitted curves can be used as the trajectory of each joint. 

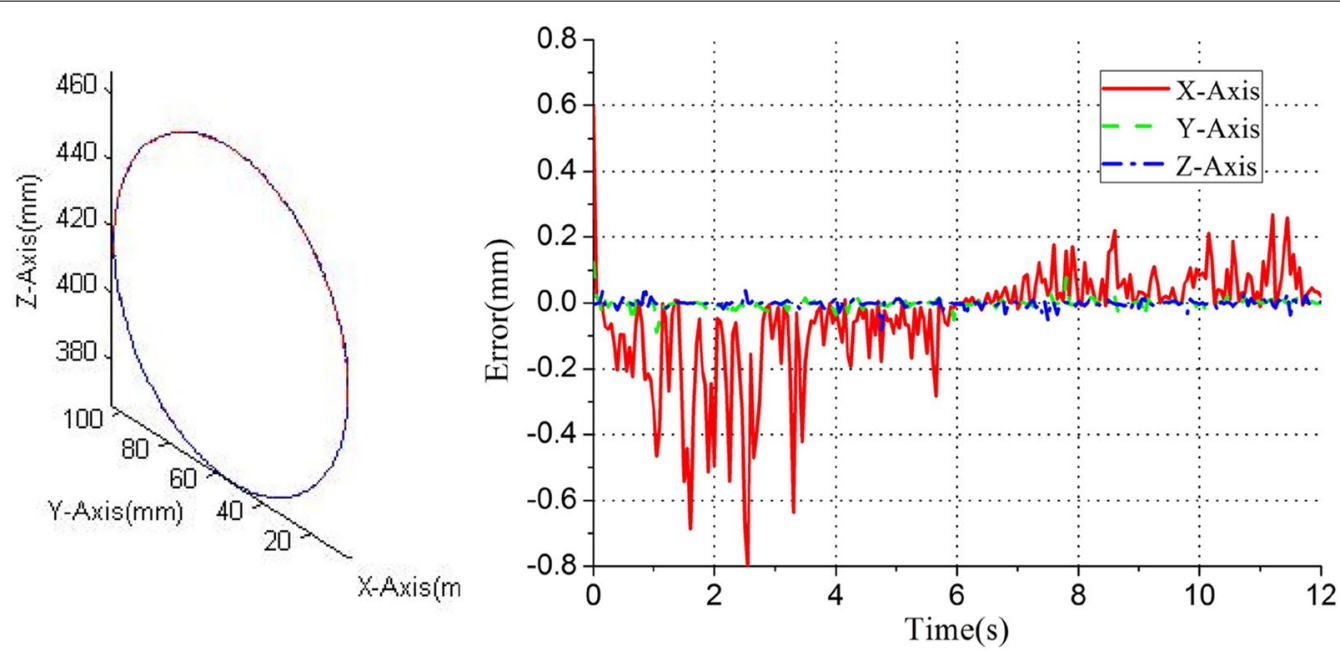

Figure 3 Ideal and actual trajectory curve of the end

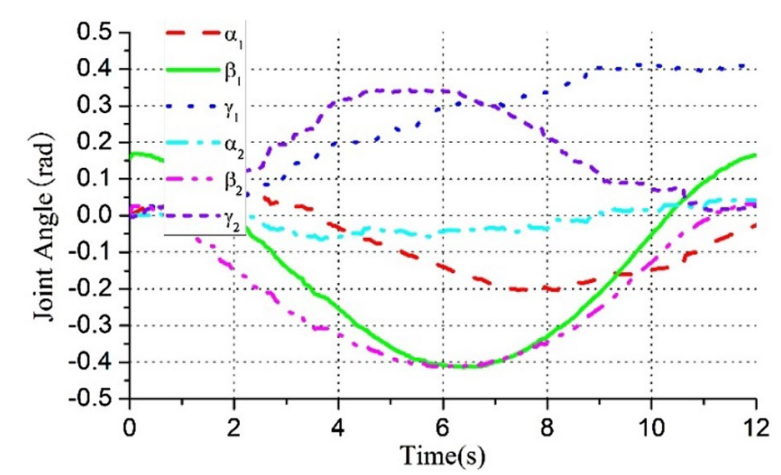

Figure 4 Joint trajectory curves

\section{Fuzzy Torque Control of Bionic Flexible Manipulator}

According to the dynamic model of the bionic flexible manipulator, the control scheme of trajectory tracking is proposed in this section. In the process of dynamic calculation, the robot model inevitably possesses structural and nonstructural uncertainty factors. Aiming at the nonlinearity of the manipulator and uncertainty of its model, the fuzzy system and computed torque method were combined to design the fuzzy torque controller.

\subsection{Computed Torque Control of Flexible Manipulator} In the control algorithm of the manipulator, the computed torque method is relatively effective. The control
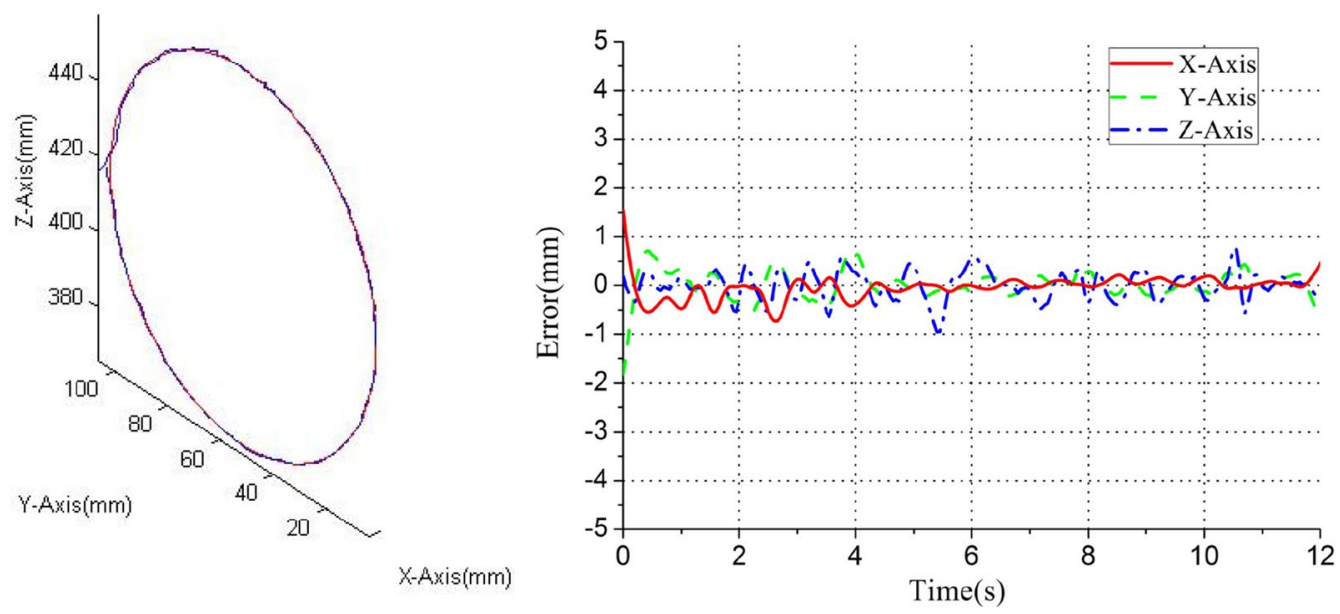

Figure 5 Ideal and fitting trajectory curve of the end 


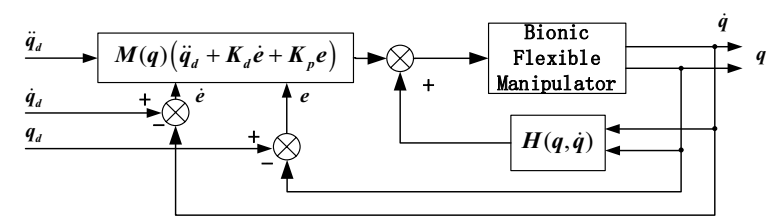

Figure 6 Block diagram for computed torque control system

idea of the computed torque method can be understood as introducing nonlinear compensation into the internal control loop of the manipulator control system. By such compensation, the manipulator can be simplified as a linear system to some extent. The computed torque method can ensure the stability of the trajectory tracking of the manipulator, and is able to control each joint separately by closed-loop control system.

\subsubsection{Computed Torque Controller}

According to the computed torque control method, a PD controller is designed in the control loop of the manipulator, which is shown in Figure 6. First introduce the control:

$$
\boldsymbol{\tau}=\boldsymbol{M}(\boldsymbol{q}) \boldsymbol{u}+\boldsymbol{H}(\boldsymbol{q}, \dot{\boldsymbol{q}}),
$$

where $\boldsymbol{H}(\boldsymbol{q}, \dot{\boldsymbol{q}})=\boldsymbol{V}(\boldsymbol{q}, \dot{\boldsymbol{q}})+\boldsymbol{G}(\boldsymbol{q})$.

According to the kinetic equation:

$$
\boldsymbol{M}(\boldsymbol{q}) \ddot{\boldsymbol{q}}+\boldsymbol{H}(\boldsymbol{q}, \dot{\boldsymbol{q}})=\boldsymbol{\tau}=\boldsymbol{M}(\boldsymbol{q}) \boldsymbol{u}+\boldsymbol{H}(\boldsymbol{q}, \dot{\boldsymbol{q}}),
$$

which is equal to

$$
M(q) \ddot{q}=M(q) u .
$$

Since $\boldsymbol{M}(\boldsymbol{q})$ is an invertible matrix, it can be obtained that

$$
\ddot{q}=u .
$$

Thus, the upper form is equivalent to the linear constant system. In the control system, the position signal can be obtained by the feedback of the system. The desired trajectory is set as $q_{\mathbf{d}}$, on this basis, PD control is introduced, which is expressed as follows:

$$
\ddot{\boldsymbol{q}}=\ddot{\boldsymbol{q}}_{\mathrm{d}}+K_{\mathrm{d}}\left(\dot{\boldsymbol{q}}_{\mathrm{d}}-\dot{q}\right)+K_{\mathrm{p}}\left(\boldsymbol{q}_{\mathrm{d}}-q\right),
$$

where, $\ddot{\boldsymbol{q}}_{\mathrm{d}}, \dot{\boldsymbol{q}}_{\mathrm{d}}, \boldsymbol{q}_{\mathrm{d}}$ represent the angular acceleration, angular velocity, and angle of each joint, respectively; and $\boldsymbol{K}_{\mathrm{p}}$ and $\boldsymbol{K}_{\mathrm{d}}$ are the PD parameters. By combining Eq. (17) and Eq. (18), the closed loop system is obtained:

$$
\ddot{\boldsymbol{e}}+K_{\mathrm{d}} \dot{\boldsymbol{e}}+K_{\mathrm{p}} \boldsymbol{e}=0,
$$

where $\ddot{\boldsymbol{e}}=\ddot{\boldsymbol{q}}_{\mathrm{d}}-\ddot{\boldsymbol{q}}, \dot{\boldsymbol{e}}=\dot{\boldsymbol{q}}_{\mathrm{d}}-\dot{\boldsymbol{q}}, \boldsymbol{e}=\boldsymbol{q}_{\mathrm{d}}-\boldsymbol{q}$. According to the controller, if the system needs to move in accordance with the desired trajectory, let $\boldsymbol{e} \rightarrow 0, \dot{\boldsymbol{e}} \rightarrow 0, \ddot{\boldsymbol{e}} \rightarrow 0$.

Substituting Eq. (18) into Eq. (14), the control law of the robot arm can be obtained:

$$
\boldsymbol{\tau}=\boldsymbol{M}(\boldsymbol{q})\left(\ddot{\boldsymbol{q}}_{\mathbf{d}}+K_{\mathbf{d}} \dot{e}+K_{\mathbf{p}} e\right)+\boldsymbol{H}(\boldsymbol{q}, \dot{\boldsymbol{q}}) .
$$

\subsubsection{Simulation Analysis of Computed Torque}

The bionic flexible manipulator is mainly driven by two parallel joints: the scapula joint and the glenohumeral joint. The expected trajectory of these two joints is $\gamma=10^{\circ} \sin \left(\frac{\pi}{2} t\right), \beta=10^{\circ} \sin \left(\frac{\pi}{2} t\right), \alpha=0^{\circ}$. The simulation control of the two joints was carried out and the control effect of the computed torque method was analyzed based on MATLAB and ADAMS.

Firstly, the proportional and differential parameters in the control loops of the scapula and glenohumeral joint are

$$
\begin{aligned}
& \boldsymbol{K}_{\mathrm{p} 1}=\left[\begin{array}{ccc}
-3750 & 0 & 0 \\
0 & -3750 & 0 \\
0 & 0 & -3750
\end{array}\right], \boldsymbol{K}_{\mathrm{d} 1}=\left[\begin{array}{ccc}
-35 & 0 & 0 \\
0 & -35 & 0 \\
0 & 0 & -35
\end{array}\right], \\
& \boldsymbol{K}_{\mathrm{p} 2}=\left[\begin{array}{ccc}
-3600 & 0 & 0 \\
0 & -1350 & 0 \\
0 & 0 & -1350
\end{array}\right], \boldsymbol{K}_{\mathrm{d} 2}=\left[\begin{array}{ccc}
-90 & 0 & 0 \\
0 & -15 & 0 \\
0 & 0 & -15
\end{array}\right] .
\end{aligned}
$$

The simulation chart of position tracking and its error can be obtained.

Through the simulation charts of the scapula joint and the glenohumeral joint in Figure 7, it was found that the computed torque method can realize the expected tracking trajectory of two joints. The maximum error of the rotation angle of the scapula joint in the $X Y Z$ direction is $\left(0.686^{\circ}, 0.531^{\circ}, 0.602^{\circ}\right)$, and the maximum error of the rotation angle of the glenohumeral joint in $X Y Z$ direction is $\left(1.107^{\circ}, 0.477^{\circ}, 0.901^{\circ}\right)$. It can be seen that the computed torque method is feasible for the tracking control of the manipulator. However, the tracking error and its range of variations are still large. The flexible manipulator is a nonlinear control system. Once the PD parameters of the control loop are determined, the control process cannot be adaptively adjusted to better control the system. Therefore, the computed torque control is improved and the fuzzy torque control method is proposed.

\subsection{Fuzzy Torque Control of Bionic Flexible Manipulator}

The manipulator control system is a typical nonlinear complex control system whose uncertain parameters change constantly in the process of movement, with some unstable disturbances. It is difficult to achieve the desired control effect for the conventional control of fixed parameters. In view of this, a control strategy is proposed in this section, which adjusts the parameters adaptively according to the control state. The fuzzy control is combined with the computed torque control to design the fuzzy torque controller. 

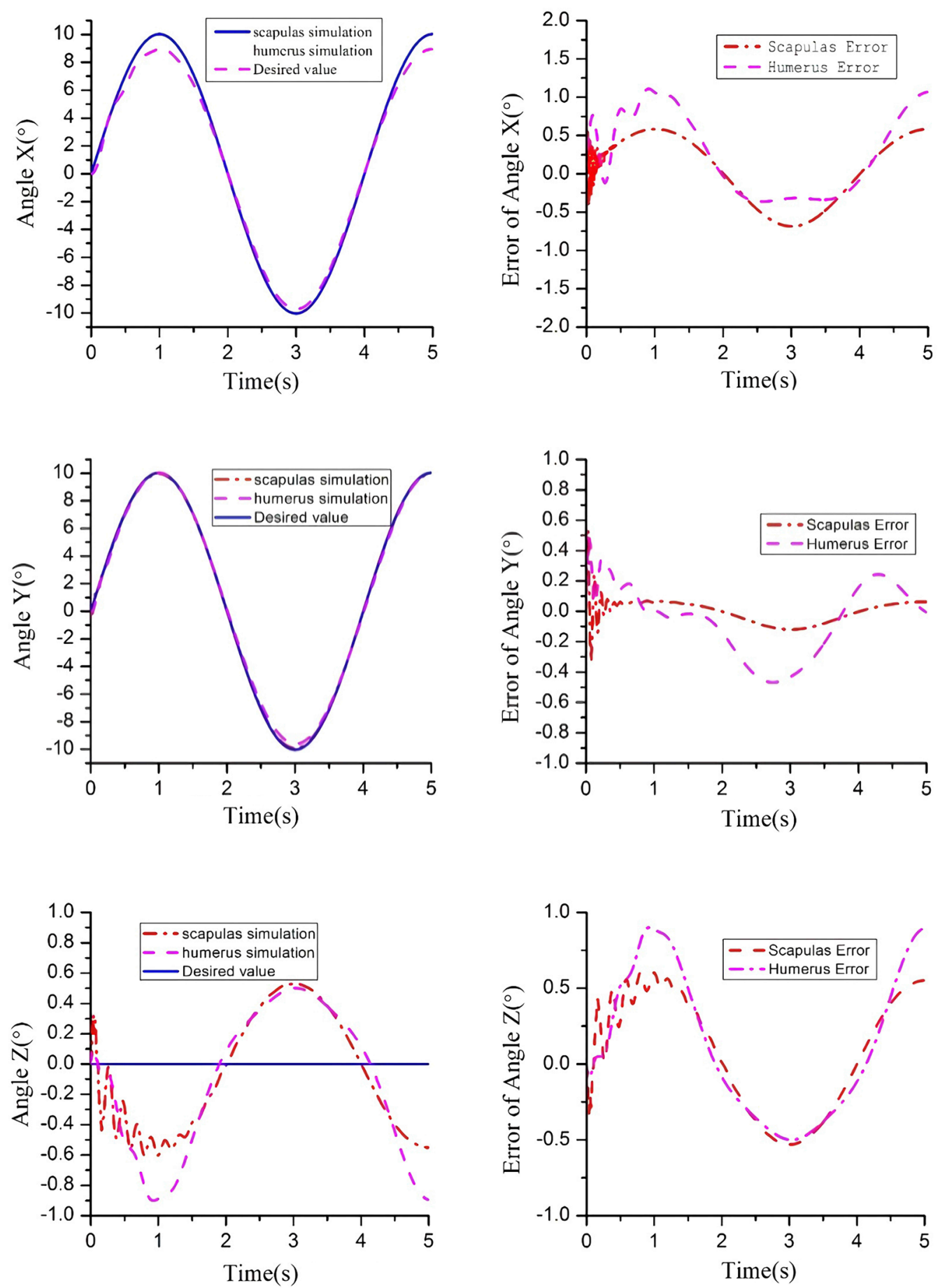

Figure 7 Simulation chart of scapula and glenohumeral joint by computed torque method

\subsubsection{Design of Fuzzy Torque Control System}

The fuzzy controller is designed to have two inputs and two outputs. According to error $\boldsymbol{e}$ and error change $\dot{\boldsymbol{e}}$ of the system real-time feedback, the PD parameters of the control loop are adjusted online in real time. The changes of $\boldsymbol{K}_{\mathrm{p}}$ and $\boldsymbol{K}_{\mathrm{d}}$ are expressed as

$$
\begin{aligned}
\Delta K_{\mathrm{p}} & =(\boldsymbol{e}, \dot{\boldsymbol{e}})_{\mathrm{p}}, \\
\Delta K_{\mathrm{d}} & =(\boldsymbol{e}, \dot{\boldsymbol{e}})_{\mathrm{d}} .
\end{aligned}
$$




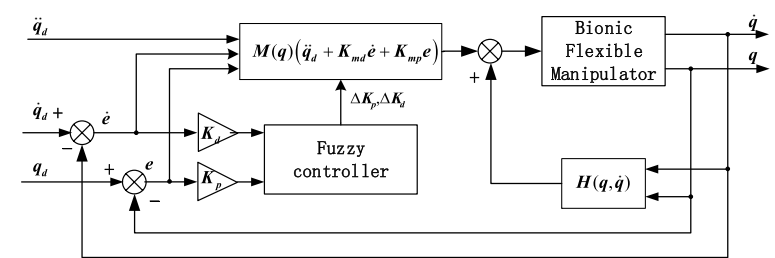

Figure 8 Block diagram for fuzzy torque control system

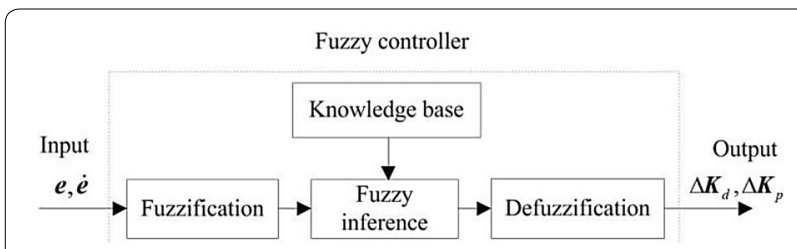

Figure 9 Block diagram of fuzzy controller

Through the upper equation, we can achieve the automatic adjustment of the PD parameters on the basis of $\boldsymbol{e}$ and $\dot{\boldsymbol{e}}$ in the control process, and realize the adjustment of the control parameters:

$$
\begin{aligned}
& K_{\mathrm{mp}}=K_{\mathrm{p}}+(\boldsymbol{e}, \dot{\boldsymbol{e}})_{\mathrm{p}}, \\
& K_{\mathrm{md}}=K_{\mathrm{d}}+(\boldsymbol{e}, \dot{\boldsymbol{e}})_{\mathrm{d}} .
\end{aligned}
$$

According to the computed torque control law above, the upper equation is substituted, and the fuzzy torque control law is obtained:

$$
\boldsymbol{\tau}=\boldsymbol{M}(\boldsymbol{q})\left(\ddot{\boldsymbol{q}}_{\mathrm{d}}+K_{\mathrm{md}} \dot{\boldsymbol{e}}+K_{\mathrm{mp}} \boldsymbol{e}\right)+\boldsymbol{H}(\boldsymbol{q}, \dot{\boldsymbol{q}}) .
$$

The PD parameters are adjusted according to the membership degree table of each fuzzy subset and the fuzzy control model of each parameter. The system develops improved adaptive ability in different environments. The block diagram of the fuzzy torque control system is shown in Figure 8.

\subsubsection{Design of Fuzzy Controller}

Fuzzy control applies linguistics to induce the operator's control strategy. Linguistic variables and fuzzy geometry theory are used to form the control algorithms. As shown in Figure 9, fuzzy control has a transformation process, including the fuzzification of precise quantity and the precision of fuzzy quantity, which mainly consist of five parts: variable definition, fuzzification, fuzzy inference, fuzzy rule, and defuzzification.

1) Variable definition
The error $\boldsymbol{e}$ and error change $\dot{\boldsymbol{e}}$ of the input variables of the controller are defined, and the output variables are $\Delta \boldsymbol{K}_{\mathrm{p}}$ and $\Delta \boldsymbol{K}_{\mathrm{d}}$.

2) Fuzzification

The discourse domain of $\boldsymbol{e}$ and $\dot{\boldsymbol{e}}$ is set as [-6,6], and that of $\Delta \boldsymbol{K}_{\mathrm{p}}$ and $\Delta \boldsymbol{K}_{\mathrm{d}}$ is [-3,3]. In fuzzy rules, the error and error rate are expressed as E and EC, respectively, and the fuzzy subsets of input and output are $\{N B$ (negtive big), $N M$ (negtive middle), $N S$ (negtive small), $Z O$ (Zero), $P S$ (positive big), $P M$ (positive middle), and $P B$ (positive small)\}. The membership function used in this section is the most common triangle membership function, which can cover all the fuzzy subsets.

$\mu(x)=\left\{\begin{array}{c}(x-a) /(b-a), \quad a \leq x \leq b \\ (x-c) /(b-c), \quad b \leq x \leq c, \\ 0, \quad \text { else. }\end{array}\right.$

3) Rule base and fuzzy inference

In the control process, the fuzzy control rules are established as shown in Table 2 and Table 3. For example, when $\boldsymbol{e}$ becomes too big, in order to accelerate the response speed and prevent integral overflow caused by $\dot{\boldsymbol{e}}$, the larger $\boldsymbol{K}_{\mathrm{p}}$ and smaller $\boldsymbol{K}_{\mathrm{d}}$ are selected. Conversely, when $\boldsymbol{e}$ becomes too small, in order to ensure a certain response speed and reduce the system overshoot, $\boldsymbol{K}_{\mathrm{p}}$ can be appropriately reduced and moderate $\boldsymbol{K}_{\mathrm{p}}$ should be selected. We can determine the corresponding fuzzy rules according to experience to realize the fast response and effective control of the system.

4) Defuzzification

The area centroid method is used for defuzzification, and the fuzzy output obtained by fuzzy inference is transformed into a precise quantity. The internal control loop of the computed torque method is improved and adjusted to realize the accurate control of the manipulator.

\subsubsection{Simulation Analysis of Fuzzy Torque}

The desired trajectory is the same as the upper section, the simulation control of the scapula joint and glenohumeral joint is implemented, respectively, and the control effect of fuzzy torque method is analyzed. The initial parameters of the proportional differential in the internal control loop of the computed torque method are the same as those of the upper section. The fuzzy torque control of the scapula joint and glenohumeral joint can be obtained, and the simulation chart of position tracking and its error is obtained as follows. 
Table 2 Fuzzy control rules of $\Delta K_{\mathrm{p}}$

\begin{tabular}{|c|c|c|c|c|c|c|c|}
\hline \multirow[t]{2}{*}{$\bar{E}$} & \multicolumn{7}{|l|}{$E C$} \\
\hline & $N B$ & $N M$ & NS & $z O$ & $P S$ & $P M$ & $P B$ \\
\hline$N B$ & $P B$ & $P B$ & $P M$ & $P M$ & PS & $Z O$ & ZO \\
\hline$N M$ & $P B$ & $P B$ & $P M$ & $P S$ & PS & 20 & NS \\
\hline NS & $P M$ & $P M$ & $P M$ & $P S$ & 20 & NS & NS \\
\hline ZO & $P M$ & $P M$ & $P S$ & $\mathrm{ZO}$ & NS & $N M$ & $N N$ \\
\hline$P S$ & PS & PS & ZO & NS & NS & $N M$ & NN \\
\hline$P M$ & PS & $Z O$ & NS & $N M$ & $N M$ & NM & $N B$ \\
\hline$P B$ & $Z O$ & 20 & $N M$ & $N M$ & $N M$ & $N B$ & $N B$ \\
\hline
\end{tabular}

Table 3 Fuzzy control rules of $\Delta K_{\mathrm{d}}$

\begin{tabular}{|c|c|c|c|c|c|c|c|}
\hline \multirow[t]{2}{*}{$E$} & \multicolumn{7}{|l|}{$E C$} \\
\hline & $N B$ & $N M$ & NS & $z O$ & $P S$ & $P M$ & $P B$ \\
\hline$N B$ & PS & NS & $N B$ & $N B$ & $N B$ & $N M$ & $P S$ \\
\hline$N M$ & PS & NS & $N B$ & $N M$ & $N M$ & NS & 20 \\
\hline NS & $Z O$ & NS & $N M$ & $N M$ & NS & NS & $Z O$ \\
\hline ZO & ZO & NS & NS & NS & NS & NS & ZO \\
\hline$P S$ & $Z O$ & $Z O$ & $Z O$ & $Z O$ & ZO & $Z O$ & $Z O$ \\
\hline$P M$ & $P B$ & NS & $P S$ & PS & PS & $P S$ & $P B$ \\
\hline$P B$ & $P B$ & $P M$ & $P M$ & $P M$ & PS & $P S$ & $P B$ \\
\hline
\end{tabular}

Observe the simulation charts in Figure 10, we can find that the fuzzy torque method can achieve the desired trajectory of two joints better than the computed torque method. The maximum error of the rotation angle of scapula joint in the $X Y Z$ direction is $\left(0.109^{\circ}, 0.260^{\circ}, 0.481^{\circ}\right)$. The maximum error of the rotation angle of glenohumeral joint in the $X Y Z$ direction is $\left(0.390^{\circ}, 0.398^{\circ}, 0.486^{\circ}\right)$. It can be seen that the fuzzy torque method has higher tracking control accuracy and the degree of shaking is significantly reduced, which is beneficial to the effective control of the physical mechanism.

\section{Test Analysis of Bionic Flexible Manipulator 4.1 Design of Test Platform}

The test system of the manipulator consists of a computer, filter, pressure relief valve, proportional valve, pneumatic pump, peripheral component interconnect digital-to-analog conversion (D/A) control card, and gyroscope. The manipulator is driven by 10 PMAs, where the scapula joint is driven by four PMAs of which the models are DMSP-10N-110-RM-CM and DMSP-10N-55-RM-CM; the maximum working pressure is $0.8 \mathrm{MPa}$. The glenohumeral joint is driven by six PMAs, of which the model is DMSP-10N-110-RM-CM; the maximum working pressure is $0.8 \mathrm{MPa}$. The pneumatic pump is the pressure source, and the maximum supplied pressure is $1.25 \mathrm{MPa}$. The control signal of the proportional valve is controlled by computer through the D/A card. Finally, the attitude data is fed back to the computer through the gyroscope, and the closedloop control is realized. The control block diagram and entity diagram of the test system are shown in Figure 11 and Figure 12, respectively.

\subsection{Results Analysis}

In order to verify the control strategy proposed in this paper, the manipulator is taken as the experimental object. The trajectory of the end of the manipulator is required to be a circle with a radius of $50 \mathrm{~mm}$, whose equation is as follows:

$$
\left\{\begin{array}{l}
p x=p x_{0}-15 \\
p y=p y_{0}+50 \cos (w t) \\
p z=p z_{0}+50 \sin (w t)
\end{array}\right.
$$

where $p x_{0}, p y_{0}, p z_{0}$ are the initial positions of the end of manipulator in the $X Y Z$ direction. According to the joint trajectory planning in Section 2.3, the scapular joint and glenohumeral joint can be precisely controlled 

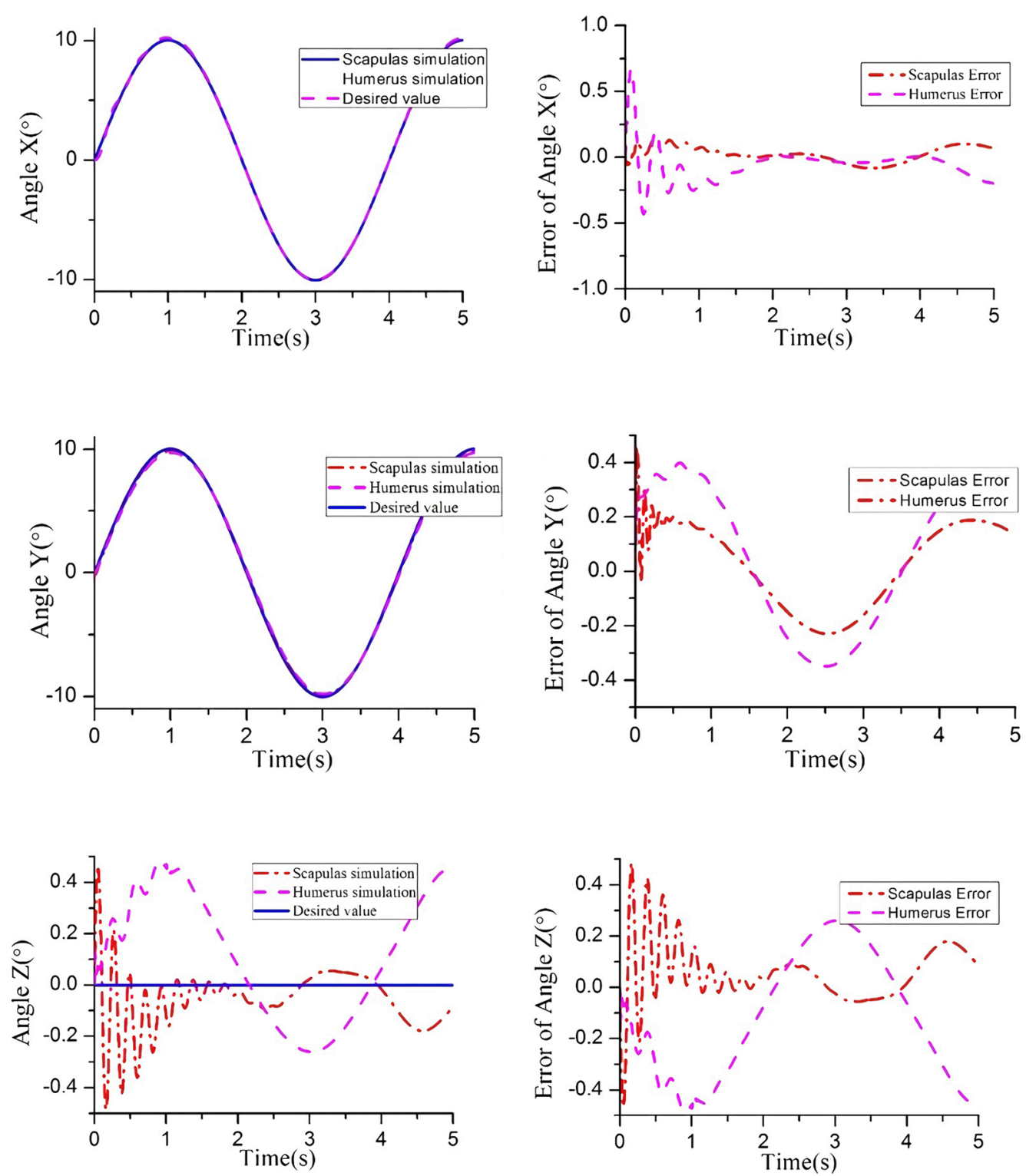

Figure 10 Simulation chart of scapula and glenohumeral joint by fuzzy torque control

by fuzzy controller to achieve trajectory tracking of the manipulator.

Experimental analysis of the control algorithm was carried out using a test platform built on the upper section. The values of the initial gain parameter of the controller are $\boldsymbol{k}_{\mathrm{p} 1}=\operatorname{diag}[-3750-3750-3750], \boldsymbol{k}_{\mathrm{d} 1}=\operatorname{diag}[-35$ $-35-35], \boldsymbol{k}_{\mathrm{p} 2}=\operatorname{diag}[-3600-1350-1350]$, and $\boldsymbol{k}_{\mathrm{d} 2}=$ $\operatorname{diag}[-90-15-15]$. The control period was set to $12 \mathrm{~s}$, and the estimation parameters of the controller are the same as those in the simulation process.
First, we used the general computed torque method to control the motion of the manipulator joint and obtain the end track tracing curve, as shown in Figure 13. The $X Y Z$ direction error range of the end position in the trajectory tracking process is $(-14.972 \mathrm{~mm}, 22.551 \mathrm{~mm})$, $(-45.421 \mathrm{~mm}, 49.912 \mathrm{~mm})$, and $(-5.790 \mathrm{~mm}, 13.388$ $\mathrm{mm}$ ) respectively. Then, we used the fuzzy torque algorithm to control the bionic flexible manipulator. Figure 14 shows that the actual motion trajectory of the end of mechanical arm is very close to the desired trajectory. The $X Y Z$ direction error range of the end position in the trajectory tracking process is $(-3.102 \mathrm{~mm}, 2.664$ 


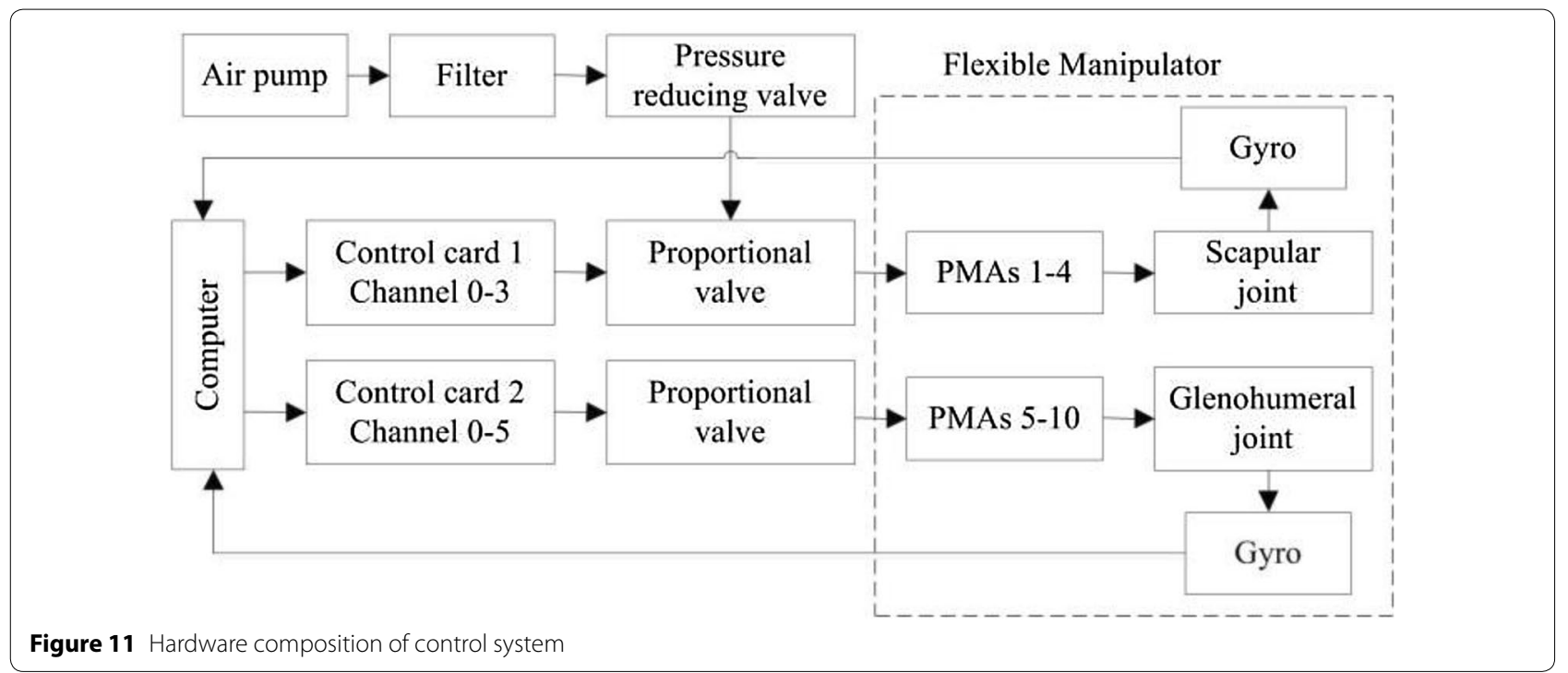

$\mathrm{mm}),(-4.693 \mathrm{~mm}, 3.411 \mathrm{~mm})$, and $(-2.034 \mathrm{~mm}, 10.114$ $\mathrm{mm}$ ). The comparison of the simulation and experiment results using the fuzzy torque algorithm are summarized in Table 4.

Compared with the computed torque method, the trajectory tracking precision of the fuzzy torque controller is much higher. However, some jitter still occurs in the process of trajectory tracking, and the experimental results are slightly larger than the simulation results, as shown in Table 4. The main reason for this phenomenon is the fluctuation in the inflation and deflation process of the proportional valve, which affects the movement process of PMA. It can be adjusted through the designed joint motion control strategy. The initial response of each joint is a step response; hence, there is large jitter at the initial time. Although the joints tend to stabilize quickly and track the desired trajectory of motion owing to the function of the fuzzy controller, the error of the tracking curve measured by experiments increases significantly compared with that in the simulation. Another reason is the error of the mathematical model of pneumatic muscle. However, experimental results show that the end of the mechanical arm can effectively track the desired trajectory; thus, the proposed fuzzy torque control algorithm is feasible.

\section{Conclusions}

(1) Based on the anatomy theory of the shoulder joint, the skeleton and muscle of the human upper arm and the mechanism of action on the skeleton in the process of movement are analyzed. The basic structure of the human upper arm was selected as a serial parallel structure.

(2) Based on the application of computed torque method in the trajectory tracking control of manipulator, a fuzzy torque control algorithm was designed. The two methods were compared by simulation, and the results show that the fuzzy torque control algorithm can compensate the error more effectively.

(3) The hardware experimental system was established. Compared with general computed torque method, the effectiveness of fuzzy torque control algorithm was verified in the actual control test of the prototype. The XYZ direction error range of the end position in the trajectory tracking process is $(-3.102 \mathrm{~mm}, 2.664 \mathrm{~mm}),(-4.693 \mathrm{~mm}, 3.411 \mathrm{~mm})$, and $(-2.034 \mathrm{~mm}, 10.114 \mathrm{~mm})$. The control study in this paper does not consider the problem of load. In the future, the load capacity and maneuverability of the manipulator will be explored in depth. 


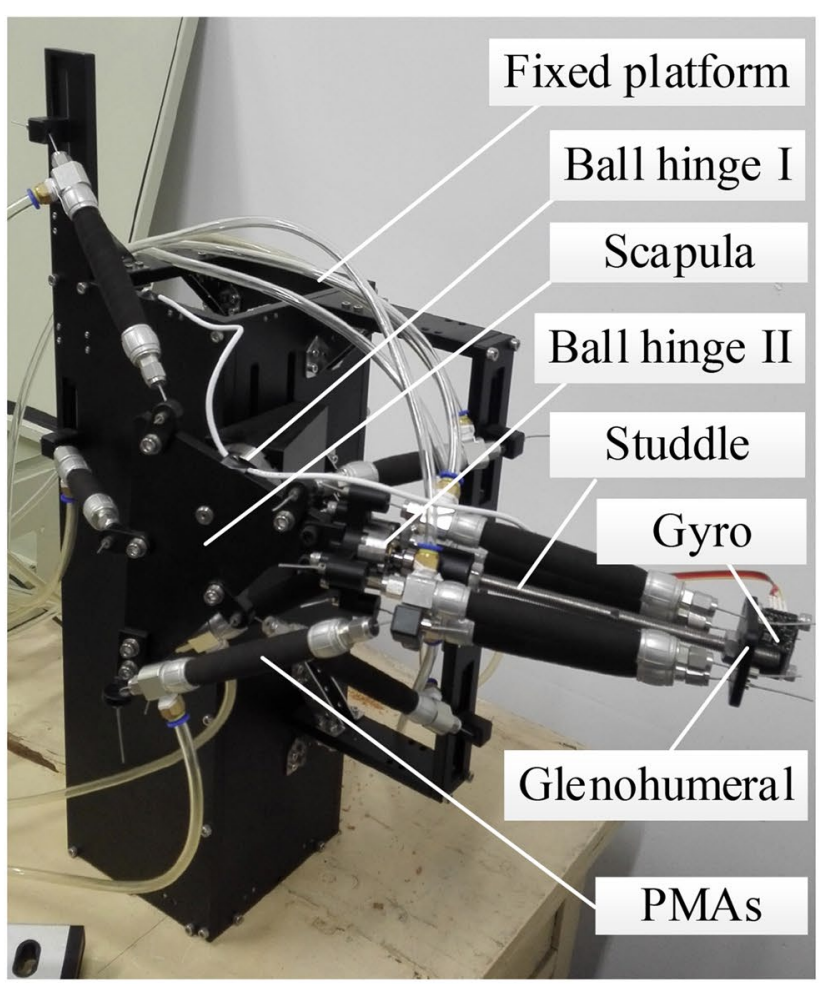

a Physical prototype

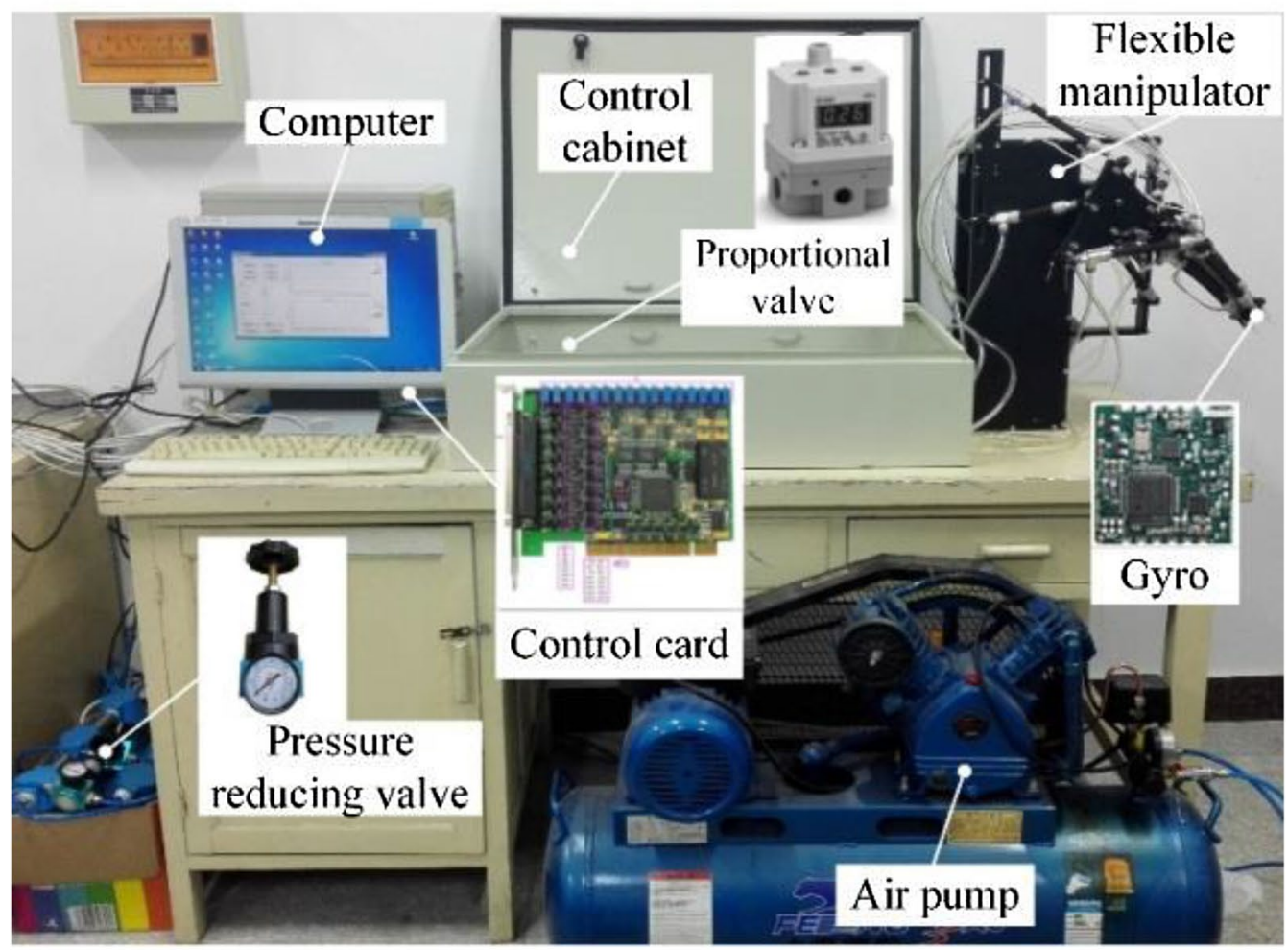

b Experimental platform

Figure 12 Test platform of the bionic flexible manipulator driven by PMAs 

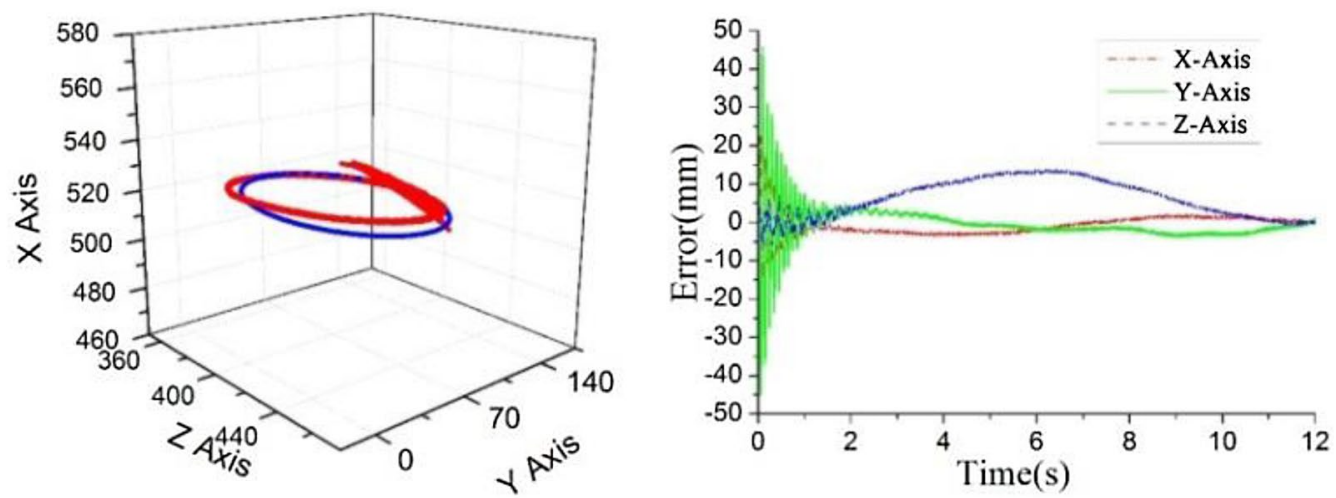

Figure 13 Circle trajectory tracking of computed torque method
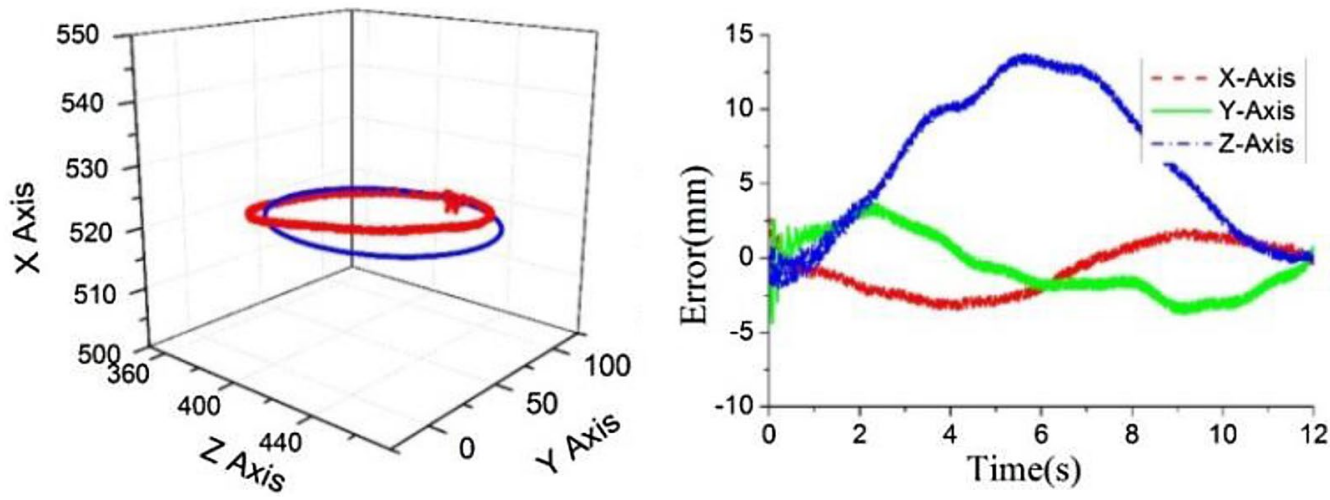

Figure 14 Circle trajectory tracking of fuzzy torque control

Table 4 Comparison of simulation and experiment using fuzzy torque algorithm

\begin{tabular}{lll}
\hline Error range & Simulation & Experiment \\
\hline$X$ direction & $-2.513 \mathrm{~mm} \sim 2.208 \mathrm{~mm}$ & $-3.102 \mathrm{~mm} \sim 2.664 \mathrm{~mm}$ \\
$Y$ direction & $-3.901 \mathrm{~mm} \sim 2.800 \mathrm{~mm}$ & $-4.693 \mathrm{~mm} \sim .411 \mathrm{~mm}$ \\
$Z$ direction & $-2.065 \mathrm{~mm} \sim 8.438 \mathrm{~mm}$ & $-2.034 \mathrm{~mm} \sim 10.114 \mathrm{~mm}$ \\
\hline
\end{tabular}

\section{Authors' contributions}

$\mathrm{KL}$ was in charge of the whole trial; $Y C$ analyzed the results and wrote the manuscript; JX designed the platforms and assisted with sampling; YW and $Y L$ assisted with the laboratory analyses and modeling; DZ contributed to the background of the study. All authors read and approved the final manuscript.

\section{Authors' Information}

Kai Liu, born in 1981, is currently an associate professor at College of Mechanical and Electrical Engineering, Nanjing University of Aeronautics and Astronautics (NUAA), China. He received his Ph.D. degree from NUAA in 2007. His main research interests include bionic robot and numerical control technology.

Yining Chen, born in 1995, is currently pursuing master degree in NUAA. She received her B.S. degrees from NUAA in 2017.

Jiaqi Xu, born in 1994, is currently pursuing master degree in NUAA. He received his B.S. degree from Nanhang Jincheng College, China, in 2015.

\section{Acknowledgements}

The authors sincerely thanks to Professor Yonghua Lu and Professor Dongbiao Zhao for critical discussion and reading during manuscript preparation.

\section{Competing Interests}

The authors declare that they have no competing interests.

\section{Funding}

Supported by National Natural Science Foundation of China (Grant No. 51405229) and Jiangsu Provincial Natural Science Foundation of China (Grant No. BK20151470).

Received: 26 August 2018 Revised: 3 June 2019 Accepted: 17 September 2019

Published online: 01 October 2019

\section{References}

[1] H Arne, M Hiroaki, I Shuhei, et al. Anthropomorphic musculoskeletal 10 degrees-of-freedom robot arm driven by pneumatic artificial muscles. Advanced Robotics, 2018: 1-14.

[2] Z Chen. Design and control of knee joint of humanoid robot based on pneumatic artificial muscles. Engineering Journal of Wuhan University, 2018, 51(1): 80-84. (in Chinese)

[3] S Xie, J Mei, H Liu. Kinematics modeling and simulation of trajectory tracking control of a foot-plate-based lower-limb rehabilitation robot. 
Journal of Tianjin University (Science and Technology), 2018, 51(5). (in (hinese)

[4] Q Zhang, C Qin, Y Sun. Dexterous hand actuated by pneumatic artificial muscle. Chinese Hydraulics \& Pneumatics, 2018, 321(5): 96-100. (in (hinese)

[5] B Guo, P Li, J Han, et al. Design of pneumatic dexterous hand control system based on data glove. Chinese Hydraulics \& Pneumatics, 2016(10). (in Chinese)

[6] P K Jamwal, S Hussain, M H Ghayesh, et al. Impedance control of an intrinsically compliant parallel ankle rehabilitation. IEEE Transactions on Industrial Electronics, 2016, 63: 3638-3647.

[7] Y Zhao, D Sun, J Chen, et al. Damping characteristics analysis of vehicular photoelectric platform with pneumatic artificial muscle. Journal of Taiyuan University of Science and Technology, 2017(1). (in Chinese)

[8] K Liu, T Ma, B Gu, et al. A new method to predict force for pneumatic muscle actuators. Advanced Robotics, 2015, 29: 1127-1136.

[9] J Lei, H Yu. Dynamics analysis of bionic flexible body driven by pneumatic artificial muscle for quadruped robot. Journal of Shanghai Jiaotong University, 2014, 48(12): 1688-1693. (in Chinese)

[10] GWang, D Chen, K Chen, et al. The current research status and development strategy on biomimetic robot. Journal of Mechanical Engineering, 2015, 51: 27-44. (in Chinese)

[11] Q Guo, Y Zhang, B G Celler. Backstepping control of electro-hydraulic system based on extended-state-observer with plant dynamics largely unknown. IEEE Transactions on Industrial Electronics, 2016.

[12] Q Guo, J Yin, Yu T, et al. Saturated adaptive control of electrohydraulic actuator with parametric uncertainty and load disturbance. IEEE Transactions on Industrial Electronics, 2017, PP(99): 1-1.

[13] Q Guo, Q Wang, Y Liu . Anti-windup control of electro-hydraulic system with load disturbance and modelling uncertainty. IEEE Transactions on Industrial Informatics, 2017, PP(99): 1-1.

[14] P Carbonell, Z P Jiang, D W Repperger. A fuzzy backstepping controller for a pneumatic muscle actuator system. IEEE International Symposium on Intelligent Control, Mexico City, Mexico, 2001: 353-358.

[15] D Meng, G Tao, A Li, et al. Adaptive robust control of pneumatic cylinders using fast switching on/off solenoid valves. Journal of Mechanical Engineering, 2015, 51: 180-188. (in Chinese)

[16] S Wei, G Shi. Hybrid position tracking control of a pneumatic artificial muscle. Journal of Shanghai Jiaotong University, 2012, 46: 201-206. (in Chinese)

[17] L Lin, J Yen, F Wang. Robust control for pneumatic muscle actuator system. Transactions of Canadian Society for Mechanical Engineering, 2013, 37: $581-590$.
[18] D X Ba, T Q Dinh, K K Ahn. An integrated intelligent nonlinear control method for a pneumatic artificial muscle. IEEE-ASME Transactions on Mechatronics, 2016, 21: 1835-1845.

[19] B Wang, B Zhang, G Shen, et al. Modeling and fuzzy control of humanoid elbow driven by cascaded pneumatic muscles. Robot, 2017, 39: 474-480. (in Chinese)

[20] L Zhao, Q Li. An active disturbance rejection control approach for one-DOF manipulator driven by pneumatic artificial muscles. Chinese Hydraulics \& Pneumatics, 2017, 3: 38-42.

[21] X Jiang, Z Wang, C Zhang, et al. Fuzzy neural network control of the rehabilitation robotic arm driven by pneumatic muscles. Industrial Robot, 2015, 42: 36-43.

[22] J Wang, X Jiang. Joint modeling and position fuzzy control of rehabilitation robot driven by pneumatic muscles actuators. Machinery, 2011, 49: 10-13. (in Chinese)

[23] Y Wei, X Li. Design and implementation of a flexible manipulator actuated by pneumatic muscle. Robot, 2005, 27: 445-449. (in Chinese)

[24] Y Liu, TWang, W Fan. Mechanism and impedance control of the ball universal joint robot driven by the pneumatic muscle actuator group. Journal of Mechanical Engineering, 2013, 49: 28-33. (in Chinese)

[25] L Wang, Y Jin, H Zhu, et al. Design and research of seven degrees of freedom robotic arm driven by pneumatic artificial muscle. Journal of Zhejiang Sci-Tech University, 2012, 29: 74-78. (in Chinese)

[26] H Yu, W Guo, HTan, et al. Design and control on antagonistic bionic joint driven by pneumatic muscles actuators. Journal of Mechanical Engineering, 2012, 48: 1-9. (in Chinese)

[27] W Zang, K Zang, H Wang. Simulation and modeling of a 4-SPS/S parallel mechanism driven by group of four pneumatic muscles. Journal of Jiamusi University (Natural Science Edition), 2016, 34(3): 350-353. (in Chinese)

[28] Y Hou, X Hu, D Zeng, et al. Biomimetic shoulder complex based on 3-PSS/S spherical parallel mechanism. Chinese Journal of Mechanical Engineering, 2015, 28: 29-37.

[29] K Liu, Z Ge, J Xu, et al. Kinematic optimization of bionic shoulder driven by pneumatic muscle actuators based on particle swarm optimization. Transactions of Nanjing University of Aeronautics \& Astronautics, 2016, 33: 301-309.

[30] A M Halder, E Itoi, K K An. Anatomy and biomechanics of the shoulder. Orthopedic Clinics of North America, 2000, 31: 159-176.

\section{Submit your manuscript to a SpringerOpen ${ }^{\circ}$ journal and benefit from:}

- Convenient online submission

- Rigorous peer review

- Open access: articles freely available online

- High visibility within the field

Retaining the copyright to your article

Submit your next manuscript at $\boldsymbol{\nabla}$ springeropen.com 